\title{
La modernización del estado en el Perú
}

\author{
State modernization in Peru \\ Juan Carlos Pasco \\ Universidad de Chile
}

\section{Introducción}

La implementación de la Gestión para resultados requiere de innovaciones que suponen esfuerzos a mediano y largo plazo, por lo que trasciende varios períodos gubernamentales. Dichas innovaciones pueden requerir de cambios en el marco legal, consensos entre funciones del Estado, capacitación de los funcionarios públicos y estructuras que fomenten la coordinación y el trabajo en conjunto (García López y García Moreno, 2010). Todo ello conlleva a procesos complejos de ensayo y error en los que intervienen varios actores por lo que es necesario establecer un ambiente político e institucional en el que los resultados sean el centro del debate público.

Como sucede en diversos países de la región, han existido diversos ciclos y esfuerzos de modernización de la administración pública en las décadas pasadas. En ese sentido, los esfuerzos por mejorar la gestión pública en el Perú han sido numerosos pero no necesariamente responden a una orientación estratégica y sostenible.

En esa línea la modernización del Estado en Perú se basó en un esfuerzo por estabilizar la economía y por reducir el gasto público, lo que obligó a reducir el tamaño del Estado. De esta manera, el esfuerzo de impulsar la Modernización recae en la Secretaría de Gestión Pública de la Presidencia del Consejo de Ministros (SGP), como ente rector del Sistema Administrativo de Modernización de la Gestión Pública a partir de Marzo de 2007. Es entonces el ente llamado a liderar el proceso de mejora en la Gestión pública y responsable de la modernización administrativa en las entidades públicas peruanas.

El último gran proceso de reforma se inició en la década pasada con la aprobación de la Ley marco de modernización de la gestión del Estado en el 2002 y con la puesta en marcha de un nuevo proceso descentralizador. En 2007 se aprueba la Ley orgánica del Poder Ejecutivo, que regula los sistemas administrativos y los sistemas funcionales para la puesta en marcha de las políticas nacionales (Llona Rosa, 2012).

*Dirección de correspondencia [Correspondence address]: Juan Carlos Pasco, Universidad de Chile E-mail: jcpasco@gmail.com
A partir del 2011, se comienzan a tomar decisiones para establecer la estrategia al 2016 y 2021, lo que llevó a elaborar documentos conceptuales y de avances realizados en materia de modernización. Estos esfuerzos fueron vistos por algunas autoridades como importantes pero que necesitaban enfoques con propuestas de acción de corto y mediano plazo. El equipo de la SGP diseñó la Estrategia de Modernización de la Gestión Pública (2012-2016) la cual permitió un mayor posicionamiento de la SGP al interior de la propia Presidencia del Consejo de Ministros del Perú (PCM). Con esa estrategia se buscó dejar el mensaje de una gestión pública que requiere mayor innovación y, a la vez, capacidades, articulación y coordinación de los distintos actores, sistemas y equipos, enfocada en el cambio y en la búsqueda de resultados de cara al ciudadano.

Asimismo, el 2011, el Perú mostró su voluntad de formar parte de la iniciativa denominada "Sociedad de Gobierno Abierto" para lo cual se comprometió a elaborar un Plan de Acción que contenga compromisos concretos en materia de transparencia y acceso a la información pública, integridad pública, gobierno electrónico y promoción del desarrollo de nuevas tecnologías, participación ciudadana y rendición de cuentas. Luego de un proceso consensuado y multistakeholder, se aprobó el "Plan de Acción del Perú para su incorporación a la Sociedad de Gobierno Abierto".

Con ello, se cuenta con un marco que permite propulsar una mayor confianza en el gobierno, eleva los niveles de cumplimiento de las políticas y asegura que las decisiones adoptadas sean percibidas como legítimas por la ciudadanía. Así, la transparencia de la información pública se ve reforzada como un principio que orienta la actividad de los funcionarios hacia una necesidad mayor de confluencia entre las actividades de producción de datos de las organizaciones estatales y las necesidades y derechos ciudadanos de un mayor conocimiento de la actividad de sus gobiernos.

Para el 2012 se formuló la Política Nacional de Modernización de la Gestión Pública (PNMGP), proceso que involucró a una serie de actores y un conjunto de esfuerzos. Se realizaron consultas a expertos nacionales e internacionales así como talleres para autoridades y funcionarios de los tres niveles de gobierno. Además, se conformó un Grupo de Trabajo Interinstitucional para la Modernización 
de la Gestión Pública (GTI) donde participaron representantes de los principales actores del proceso de modernización quienes revisaron los avances del equipo de la SGP y del equipo especialista externo.

Es así que, en Enero de 2013, se aprobó la PNMGP, la cual busca ser el "principal instrumento orientador de la modernización de la gestión pública en el Perú, que establece la visión, los principios y lineamientos para una actuación coherente y eficaz del sector público, al servicio de los ciudadanos y el desarrollo del país" (Secretaría de Gestión Pública de la Presidencia del Consejo de Ministros, 2013).

Esta propuesta de política pública fue profundizada en el Plan de Implementación donde se definieron las acciones, metas, indicadores, plazos y entidades públicas responsables de la implementación de los objetivos y lineamientos de la PNMGP. Además, este documento generó dos nuevas herramientas/instrumentos para apoyar al proceso: la Gestión de iniciativas de modernización, para implantar una cultura de mejora constante, progresiva y continua de los servicios públicos enfocados en el ciudadano; y el desarrollo de Mecanismos de incentivo y apoyo a los esfuerzos de modernización de la gestión en todos los organismos públicos y niveles de gobierno.

\section{Componentes de una gestión pública orientada a resultados en el Perú}

La gestión orientada a resultados al servicio del ciudadano es un cambio decisivo que implica superar el tradicional enfoque de organizar los servicios por la oferta, aquello que el Estado estima que puede proveer, por un enfoque de demanda, que considera lo que la ciudadanía estima como necesario.

El modelo peruano se basa en cinco pilares y tres ejes transversales que coadyuvan a la gestión integral de los mismos. Los Pilares son: a) Alineamiento de Políticas Públicas, Planes Estratégicos y Operativos, b) Presupuesto para Resultados, c) Gestión por procesos, simplificación administrativa y organización institucional, d) Servicio Civil meritocrático y, e) Seguimiento, evaluación y gestión del conocimiento. Y los tres ejes transversales son: a) Gobierno Abierto, b) Gobierno electrónico y, c) Articulación Intersectorial.

De esta manera, se rescata el valor y funcionalidad de las políticas públicas en cuanto integran y dan coherencia a la intervención del Estado; el planeamiento estratégico remarca la responsabili- dad de cada institución por atender los temas del entorno estableciendo las prioridades de acción y reflejándolas en los Planes operativos. Por su parte, con la vigencia del presupuesto para resultados se garantiza que los presupuestos sean asignados en función a los productos con los cuales se satisface las demandas ciudadanas. Esto dentro del equilibrio fiscal y la articulación de la acción pública en el territorio.

La gestión por procesos, realza la importancia de las "cadenas de valor" en tanto permiten asegurar que los bienes y servicios produzcan resultados. Los recursos esenciales para optimizar la cadena de valor son el personal, la infraestructura, los equipos, las técnicas y métodos, y las finanzas. El servicio civil meritocrático, refleja el esfuerzo por visibilizar la importancia de los recursos humanos para el cumplimiento de los objetivos y el logro de los resultados de cada entidad. El seguimiento, evaluación y gestión del conocimiento, prioriza la medición con transparencia, eficacia en el cumplimiento de los objetivos, la eficiencia en el uso de recursos y la calidad o el grado de satisfacción percibida por los ciudadanos atendidos. La gestión del conocimiento pone de relieve la funcionalidad de la administración de los aprendizajes organizacionales para mejorar el funcionamiento de las entidades.

Sobre los ejes transversales, el Gobierno Abierto expresa la necesidad de continuar con los esfuerzos por garantizar y promover la transparencia, la participación ciudadana, la integridad pública, haciendo uso de la tecnología para elevar los niveles de eficacia y garantizar la rendición de cuentas. El Gobierno Electrónico, se conceptualiza como una herramienta fundamental para modernizar la gestión pública en cuanto complementa y acompaña la gestión por procesos, apoya el seguimiento y evaluación e impulsa el gobierno abierto. La Articulación Interinstitucional pone el acento en dos ejes de relación interinstitucional, el vertical: entre entidades de distintos niveles de gobierno, nacional, regional y local; y el horizontal: entre entidades de un mismo nivel de gobierno, en el nivel nacional entre sectores, y a nivel descentralizado, entre gobiernos regionales y locales.

De no institucionalizarse, de no lograr el compromiso de los diversos actores y en tanto la SGP no asegurase la solvencia técnica y el apoyo político para posicionar a la Modernización dentro de la Agenda Pública, todo lo avanzado será insuficiente. 


\section{Avances en la implementación}

Vale la pena comenzar a identificar cuáles fueron los avances logrados así como las acciones que se realizaron en el período 2011 a mayo del 2014 .

Alineamiento de Políticas Públicas, Planes Estratégicos y Operativos: En este aspecto el CEPLAN ha ido desarrollando propuestas normativas para orientar las acciones de diseño y alineamientos de Planes de Desarrollo. Sin embargo el mayor avance y reto asumido ha sido el diseño, validación y aprobación de la "Directiva General del Proceso de Planeamiento Estratégico - Sistema Nacional de Planeamiento Estratégico" publicada el 2013 que tiene el objetivo de establecer los principios, normas, procedimientos e instrumentos del Proceso de Planeamiento Estratégico en el marco del Sistema Nacional de Planeamiento Estratégico - SINAPLAN.

Presupuesto para Resultados: Este proceso ha antecedido al proceso de modernización descrito porque, en el país, se viene implementando desde el 2007. Según información publicada en el Portal del Ministerio de Economía y Finanzas (MEF) el $62,1 \%$ del presupuesto tiene un enfoque hacia resultados el año 2014 y de los 73 Programas Presupuestales diseñados bajo este enfoque, 40 cuentan con un sistema de indicadores de desempeño de resultados y productos. Más allá de los compromisos adquiridos por el MEF en el Plan de Implementación de la PNMGP, es importante destacar que la SGP el 2011 diseñó el Programa Presupuestal "Programa Articulado de Modernización de la Gestión Pública" y que ha ido sufriendo mejoras en cada nueva edición.

Gestión por procesos, simplificación administrativa y organización institucional: Desde el año 1977 que las entidades no contaban con un documento actualizado a las realidades y avances metodológicos y tecnológicos para implementar procesos. Ante ello la SGP desarrolló, durante el segundo semestre del 2013 y hasta febrero de 2014, un nuevo marco normativo y metodológico para la implementación de la Gestión por Procesos en las entidades del Estado de los tres niveles de gobierno (nacional, regional y local), sobre la base de un diagnóstico respecto al grado de avance de la Gestión por Procesos en las entidades del Sector Público Peruano.

Dentro de este marco de procesos, otro avance logrado fue la implementación y posterior seguimiento a los resultados del Concurso "El Trámite de Más". Este mecanismo de participación ciudadana implementado por la SGP, buscaba incorporar la voz ciudadana en las propuestas de desburocratización del Estado a partir de su participación en la determinación de los trámites a simplificar, estandarizar, eliminar, o automatizar. Las vías para participar fueron cuatro: vía web, correo electrónico, teléfono y buzón postal. Se tuvieron 3,970 postulaciones, siendo seis los trámites ganadores seleccionados a través de un jurado constituido por personalidades de la sociedad civil, medios de comunicación y voceros de opinión en temas públicos.

Por otra parte, durante estos años se continuó impulsando la implementación de la estrategia MAC "Mejor Atención al Ciudadano" que comenzó el 2011. Aquí se han integrado un conjunto de entidades a nivel nacional, regional, local; para brindar a los ciudadanos la posibilidad de realizar diversos trámites en un único lugar, ofreciendo un servicio personalizado y de calidad, ahorrándole tiempo y recursos. Adicionalmente, la SGP desde el 2013 en adelante, apoya en la coordinación interinstitucional a fin de llevar los servicios del Estado a la zona rural Amazónica a través de la implementación de una estrategia que incluye la implementación de las Plataformas Itinerantes de Acción Social.

También, se continuaron y fortalecieron esfuerzos en torno al ordenamiento de la estructura del Estado Peruano y se aprobó el Registro Unificado de Entidades del Estado Peruano (RUEEP), sistema que permite registrar a todas las entidades públicas del Estado a fin de contar con un único repositorio donde todos los demás sistemas se articulen. El RUEEP además cuenta con un Código Único de Entidad (CUE) que permitirá (a modo de código interbancario) tener un mismo listado en todos los sistemas públicos.

Servicio Civil meritocrático: Es SERVIR el ente rector del Sistema Administrativo de Recursos Humanos en el país y tiene como responsabilidad impulsar la reforma del Servicio Civil. En la actualidad SERVIR, que como entidad existe desde Junio de 2008, ha venido impulsando cambios en el ámbito de los Recursos Humanos, siendo de los más recientes y de mayor trascendencia la aprobación de la Ley № 30057 del Servicio Civil y su Reglamento de reciente aprobación. Esta ley busca establecer un régimen único para todas las personas que prestan servicios al Estado y comprende a todos los servidores civiles, independiente de su nivel de gobierno.

Con ello, se desarrolló una propuesta de intervención conjunta de manera que los enfoques de procesos y gestión de puestos se implementarán en las entidades que vayan entrando a la Ley del Servicio Civil. Además, como parte del diseño del Sistema Administrativo de Modernización de la SGP se estuvo trabajando con SERVIR en el diagnóstico de capacidades de los servidores a cargo del sistema de modernización en las entidades, a fin de medir 
los conocimientos de los operadores del sistema cuyo resultado será un insumo para la definición de perfiles de puestos y funciones que toda entidad debería contar.

Seguimiento, evaluación y gestión del conocimiento: La SGP desde el diseño de su Estrategia de Modernización de la Gestión Pública se había propuesto desarrollar los mecanismos de seguimiento y difusión de la modernización, pretendiendo ser un espacio de apoyo al cambio en la gestión pública mediante el seguimiento y evaluación del proceso de modernización en los tres niveles de gobierno. Además se busca retroalimentar a las políticas y lineamientos técnico administrativos de manera que estén acordes con las necesidades del país (Pasco, 2014).

Por otra parte, la SGP constituyó un equipo ad hoc para esta temática, creándose así la Coordinación de Seguimiento, Evaluación y Difusión de Políticas de Modernización del Estado. Una de las primeras acciones y avances que se lograron a partir de ello fue la "Elaboración de Línea de Base de los lineamientos de la PNMGP, incluyendo su componente de simplificación y los indicadores del plan de gobierno abierto" que se llevó adelante entre Septiembre de 2013 y Abril de 2014. A la fecha, se espera que este documento pueda ser publicado por la SGP para que sume en la construcción de información sobre Modernización en el país.

Esta coordinación además elaboró un primer informe de seguimiento semestral al Plan Nacional de Simplificación Administrativa; ii) Reporte semestral de seguimiento al Plan de Acción de Gobierno Abierto; iii) Reporte de Seguimiento al Manual Para Mejorarla Atención a la Ciudadanía en las Entidades de la Administración Pública y; iv) Reportes Trimestrales del 2013 en cuanto a Portales de Transparencia Estándar.

Gobierno Abierto: El primer avance a considerar es la aprobación del Primer Plan de Gobierno Abierto 2012-2014, el cual contiene compromisos concretos del Estado Peruano sobre: a) Transparencia y acceso a la información, b) Integridad pública, d) participación ciudadana y rendición de cuentas y e) gobierno electrónico para mejorar los servicios a la ciudadanía. Meses después se conformó la Comisión Multisectorial de carácter permanente compuesta por el Poder Ejecutivo, Poder Judicial, Sociedad Civil y gremios empresariales, encargada de realizar el seguimiento a las metas propuestas en el referido Plan de Acción. Con este Plan el Perú pasó a formar parte de la Sociedad de Gobierno Abierto internacional. Por otra parte, se crearon los mecanismos de seguimiento del Plan y se realizó un informe evaluación de los avances del Plan de cara al primer año de aprobado el mismo -por su parte la Sociedad de Gobierno Abierto Internacional -OGP- ha presentado su Mecanismo de Revisión Independiente 2012-2013-. Finalmente, al momento de elaborar el presente documento, la SGP en su calidad de secretaría técnica de la Comisión estaba culminando el proceso de elaboración del II Plan AGA 2014-2016, realizando un diseño participativo con talleres y reuniones de trabajo a nivel regional (Ayacucho, Piura, San Martín y dos en Lima), con una participación de más de 380 asistentes entre funcionarios, representantes de la sociedad civil, academia y sector empresarial.

Gobierno electrónico: En este espacio, la entidad líder es la Oficina Nacional de Gobierno Electrónico e Informática (ONGEI), Órgano Técnico Especializado que depende directamente del Despacho de la Presidencia del Consejo de Ministros (PCM). En estos años la ONGEI ha comenzado a impulsar los temas de interoperabilidad como enfoque de articulación interinstitucional de cara a mejores servicios públicos y gestión de la información del Estado y, además, ha ido desarrollando instrumentos para el desarrollo de las actividades de supervisión en el despliegue de proyectos TIC en el Estado, como la Política Nacional de Gobierno Electrónico que se suma a otros ya existentes como el Plan Estratégico de Gobierno Electrónico (PEGE).

Articulación Intersectorial: Más allá que en el Perú existen diversas comisiones y mecanismos de articulación inter e intra gubernamentales, de cara a lo que compete a impulsar el proceso de modernización, este es un eje aún por trabajar y se requerirá la conformación del Grupo de Trabajo Interinstitucional (GTI) que se constituya como mecanismo para articular a los sistemas administrativos.

\section{Un aporte adicional: los porta- les de transparencia estándar}

Las organizaciones son sistemas que producen conocimiento a través del entramado de tareas de diversa complejidad. La naturaleza de esta complejidad se halla atravesada por los problemas relativos a cuan explícito es el conocimiento que emplean. La actividad de los individuos que la conforman está directamente relacionada a su experiencia personal frente a sus tareas así como al carácter del propósito de las mismas. Con frecuencia, las personas desarrollan tareas que requieren un elevado nivel de formalización de sus resultados, como procesos administrativos cerrados o la ejecución de cadena de tareas productivas mecanizadas (López Egaña, 2014). 
Por tanto, la información producida por las organizaciones en un contexto de gobierno abierto nos conduce a señalar cómo la naturaleza de la misma produce consecuencias en las relaciones entre Estado y ciudadanos. Si entendemos como principio que la información produce un perfil de las organizaciones y afecta el comportamiento tanto de las mismas como de las personas que la conforman, es coherente extrapolar también los efectos hacia los receptores externos de esos efectos; es este caso, los ciudadanos. La condición inherente a la realización de un esquema de gobierno abierto es la posibilidad de hacer de la información un instrumento que acreciente los niveles de desarrollo de actitudes democráticas y que estas retroalimenten el diseño del Estado hacia procesos que coadyuven a un mayor y más amplio ejercicio de derechos ciudadanos.

Los portales de transparencia estándar son un medio uniforme y estabilizado de difusión de información pública que promueve acciones de transparencia activa en las organizaciones del sector público desde el año 2010. Los portales de transparencia estándar permiten, por una parte, a los funcionarios actualizar la información que deben mostrar a los ciudadanos, y, por otra, establecer una relación de buena fe con los ciudadanos interesados en saber cuándo y de qué modos se lleva a cabo la gestión de los asuntos públicos. Estos portales están concebidos como nodos de promoción de información de las organizaciones del sector público de modo que hagan posibles mayores niveles de comunicación con los ciudadanos. La Secretaría de Gestión Pública, es la instancia encargada de impulsar la dirección de las políticas relativas a la transparencia y acceso a la información pública.

Esto incrementaría los niveles de gobernabilidad y la extensión de prácticas democráticas en la sociedad. La modernización del Estado se comprende dentro de un esquema de gobierno abierto, el cual señala un paradigma nuevo de ejercicio del derecho de acceso a la información, y una nueva valoración de la información como vínculo entre ciudadanos y Estado.

Por ello, la implementación de los portales de transparencia estándar contienen formatos estabilizados mediante los cuales las administraciones públicas registrarán y actualizarán información de gestión, orientándose fundamentalmente hacia el esquema organizacional, procedimientos, normas marco, comunicaciones oficiales, información presupuestal y del personal e indicadores de desempeño.

En este marco, la SGP desarrolló una guía para la evaluación y el cumplimiento de las directivas sobre transparencia mediante los portales estandarizados para este propósito. Aunque se han presen- tado frecuentes informes sobre el cumplimiento de las organizaciones públicas en el uso del portal de transparencia estándar, aún queda pendiente saber la dinámica interna que producen en los funcionarios y la actividad administrativa en general. Dado que es una práctica reciente en el Estado, no se conoce el comportamiento de los funcionaros en relación a la transparencia y el tipo de nociones que existen dentro de las organizaciones acerca del valor de la información que producen y la naturaleza del ejercicio del derecho al acceso a la información de los ciudadanos (Catlaw y Sandberg, 2014).

Entonces, los portales estandarizados pueden concebirse como instrumentos de gran valor para la difusión pública de contenidos de la actividad de las instituciones del Estado; pero también puede llegar a observarse como instrumentos de carácter burocrático, tanto desde los propios funcionarios como desde la mirada de los ciudadanos. Esto lleva a reflexionar sobre qué consecuencias trae comprender el mandato del acceso a la información y qué tipo de actitudes producen tanto en las organizaciones, funcionarios y ciudadanos (Cooper et al., 2006). Los portales de transparencia estándar promueven cierto patrón de actitudes hacia la información que aún no han sido suficientemente comprendidos y que deben ser analizados para dimensionar la escala de efectos que produce movilizar la información de la actividad de las organizaciones públicas.

El objeto de eliminar e inhibir las prácticas de corrupción en el aparato público es promover una administración que no esté bajo el arbitrio de los intereses privados sino en los intereses concertados de la sociedad en su conjunto. La simplificación administrativa y la reforma general de la administración pública se enfrentan continuamente al reto de poder inhibir las prácticas de corrupción haciendo un balance entre la suficiente supervisión y la eficiencia de la cadena de procesos administrativos.

Una respuesta inteligente a este dilema es cambiar la percepción que existe acerca de la información pública. El proceso de combatir los actos de corrupción ha centrado la atención en la información como un instrumento de vigilancia, sin embargo es pertinente platear la necesidad de repensar cómo es posible hacer la información pública un elemento de afianzamiento de la acción pública y un insumo para mejorar las actitudes cívicas y cotidianas de los ciudadanos.

La transparencia activa debe extender su naturaleza y amplificar la noción de utilidad de la información para enlazar los requerimientos cotidianos de información de los ciudadanos y refigurar la acción estatal de modo que haga posible hacer de la producción de información un elemento central de 
sus operaciones. Por lo general, la información de los portales de transparencia estándar es percibida como un producto residual que, con una frecuencia variable de tiempo, es suministrada al público; esta información debería alimentar la capacidad de orientación de los ciudadanos en sus propias localidades para promover acciones individuales y colectivas que permitan mejorar la calidad de vida a partir de un ejercicio informado de sus acciones.

Podemos decir entonces que los portales de transparencia estándar aún tienen un capítulo a desarrollar en base a esos elementos. Esto lleva a reflexionar si los portales de transparencia estándar son la herramienta adecuada para este tipo de información o se requiere un instrumento convergente para hacer de la información pública de servicios un medio de decisiones ciudadanas cotidianas. Creemos que es importante abrir el debate sobre la naturaleza de la información, y en general, de las plataformas de comunicación de información de la administración pública para enfrentar esta coyuntura. También creemos que los gobiernos locales ofrecen una oportunidad importante para comprender estos asuntos y tratarlos como fenómenos de naturaleza emergente; es decir, los gobiernos locales pueden mostrar con un mejor grado de pertinencia sobre estas consideraciones y llevarlas a extenderse sobre el total de los actos de producción de información de las estructuras estatales.

La administración pública entiende que el mandato de la ley de transparencia los conmina a establecer los portales de transparencia estándar en los sitios web de sus organizaciones. Esto puede haber contribuido a que los ciudadanos entiendan la información publicada como un elemento de características administrativas, contenido burocrático y de poco valor cotidiano. En general los portales web de las instituciones son el nodo central de las plataformas de comunicación, donde se concentran información de diversa magnitud, utilidad y diverso auditorio.

Interpelar la visión de qué tipo de información producen las organizaciones públicas para ofrecer a los ciudadanos y qué tipo de consecuencias tienen el actual tipo de información ofrecida es trascendental para lograr una convergencia armónica entre el valor de la información como una forma de experiencia de los asuntos públicos y la reforma y modernización de la administración del Estado desde la comprensión de las necesidades de información de las personas para el ejercicio de sus derechos.

Es así que los portales de transparencia estándar cumplen un papel importante en la reforma de la administración pública y traducen los ideales y principios de gobierno abierto. Las características de gobiernos abiertos que cimientan su relación con la sociedad a través de la información son connaturales a la naturaleza misma de la información que hacen accesible a los ciudadanos. De todos los modos la información es puesta en valor, el poder de su difusión cataliza las intenciones de promover un comportamiento cívico que enfatice la participación desde el conocimiento del funcionamiento del Estado, tanto en lo que respecta a sus procederes en el cumplimiento de su mandato como en la resolución del ejercicio de derechos.

\section{Recomendaciones}

La PNMGP tiene poco más de año y medio, poco tiempo como para asegurar que generará los cambios que se requieren en la calidad de servicios así como coadyuve a la construcción e implementación del Estado moderno al servicio de las personas. A continuación, me permito plantear once recomendaciones que la SGP, desde mi experiencia, deberá abordar o profundizar a futuro:

Se tiene que asegurar que las nuevas herramientas y mecanismos planteados por el Plan de Implementación de la PNMGP se logren. Ello hace imperativo que se avance en el: i) Desarrollo normativo e implementación del Sistema de Gestión de iniciativas de modernización y ii) Diseño e implementación de mecanismo de incentivos para la modernización en el Poder Ejecutivo.

Se tiene que institucionalizar el Sistema Administrativo de Modernización del Estado por lo que se debe desarrollar una propuesta de qué funciones y perfiles de profesionales deben encargarse de impulsar la mejora en su gestión.

El entendimiento alrededor a modernización de la gestión pública en las entidades del país es muy heterogéneo. De allí que sería interesante que la SGP apruebe un documento orientador para entidades de los tres niveles de gobierno (o bien diferenciado por entidades rectoras de sistemas administrativos, de sistemas funcionales y entidades en general).

Aunque no ha sido tocado en este documento, existen diversas iniciativas que ha impulsado la SGP desde años anteriores en temas (simplificación de trámites, mejor atención al ciudadano, etc.) que son necesarios de reforzar.

A nivel de propuestas innovadoras, aparece como necesaria la Implementación de la gestión por procesos a nivel nacional, como herramienta de apoyo a gestión interna, el logro de resultados y como herramienta que coadyuva además al proceso de implementación del servicio civil meritocrático peruano. 
Apuntalar los esfuerzos en torno al seguimiento y posterior evaluación del proceso de modernización. Para ello, se debe asegurar la implementación del aplicativo informático que viene siendo construido por la SGP ello implicará seguramente la construcción de estrategias diferencias dada la heterogeneidad en temas de capacidades técnicas y tecnológicas en el país. Adicionalmente, se debe trabajar coordinadamente con entidades como el MEF y CEPLAN para construir e implementar un modelo técnico/normativo que soporte iniciativas mayores de gestión de indicadores, metas y resultados direccionados a los objetivos del país y también del gobierno de turno.

Construir una propuesta de implementación de gestión del conocimiento en las entidades públicas. Una posibilidad es que se empiece por espacios más conocidos y valorados como el de sistematización y difusión de Buenas Prácticas en Gestión Pública. Si ello es impulsando de manera sistemática se podrá construir un repositorio de experiencias y conocimientos públicos que sería en sí mismo un logro como país. Otro espacio que se recomienda es construir una comunidad de práctica que reúna a los equipos especialistas en modernización que se definan en las entidades públicas y, que este espacio, se convierta en un foco de aprendizajes y sistematización de conocimiento en el tema -así como piloto para probar propuestas en torno a la gestión del conocimiento que se conviertan en insumos para una futura propuesta técnica y normativa de implementación de este tema en el país.

En temas de Articulación intersectorial, es necesario reiterar necesidad de conformación del Grupo de Trabajo Interinstitucional (GTI) que se constituya como mecanismo para articular a los sistemas administrativos. Ello además se encuentra como una meta del Plan de Implementación de la PNMGP.

En temas de Gobierno Abierto, una vez que se apruebe la segunda versión del Plan de Acción de Gobierno Abierto, se deberá apoyar -con recursos, acciones de difusión, grupos de trabajo, informes de seguimiento, etc.- a la Comisión Multisectorial de carácter permanente para seguimiento del gobierno abierto.

La propia idea de transparencia nos refiere a una metáfora de muros de cristal. Durante mucho tiempo los estados han desarrollado sus actividades como si fuesen castillos amurallados. Si bien los actos de gobierno y de gestión provienen en una democracia del mandato ciudadano, estas aparecen ante aquellos como una actividad oscura y ajena a la existencia cotidiana.

Es preciso extender la noción de transparencia a un ámbito mayor de cuestiones relativas a la información pública. Si el propósito y objeto fundamental de las iniciativas de transparencia es mejorar las relaciones entre Estado y ciudadanos, debemos comprometer la investigación sobre este asunto a indagar sobre cuáles son las acciones y expectativas que vinculan a los ciudadanos más directamente con el Estado.

\section{Referencias}

Catlaw, T. J. y Sandberg, B. (2014). Â Government $\hat{A} \gg$ Info-Liberalism, Active Citizenship, and the Open Government Directive. Administration \& Society, 46(3):223-254.

Cooper, T. L., Bryer, T. A., y Meek, J. W. (2006). Citizen-Centered Collaborative Public Management. Public Administration Review, 6(66):7688.

García López, R. y García Moreno, M. (2010). La Gestión para Resultados en el Desarrollo. Avances y Desafíos en América Latina y el Caribe. Banco Interamericano de Desarrollo, Washington, DC.

Llona Rosa, M. (2012). Hacia una gestión pública que impacte en el ciudadano. Documento del XVII Congreso Internacional del CLAD sobre la Reforma del Estado y de la Administración Pública, Cartagena de Indias. 